Revue internationale de l'économie sociale

Recma

Repenser l'économie

Rethinking the economy

Jean-François Draperi

Numéro 310, octobre 2008

URI : https://id.erudit.org/iderudit/1021097ar

DOI : https://doi.org/10.7202/1021097ar

Aller au sommaire du numéro

Éditeur(s)

Association Recma

ISSN

1626-1682 (imprimé)

2261-2599 (numérique)

Découvrir la revue

Citer ce document

Draperi, J.-F. (2008). Repenser l'économie. Revue internationale de l'économie sociale, (310), 4-5. https://doi.org/10.7202/1021097ar d'utilisation que vous pouvez consulter en ligne.

https://apropos.erudit.org/fr/usagers/politique-dutilisation/ 


\section{REPENSER L'ÉCONOMIE}

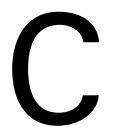

e numéro 310 parait dans un contexte économique très particulier, marqué par une crise financière qui ébranle le bien-fondé du capitalisme patrimonial et met en lumière les dangers d'un retrait quasi total des Etats du champ de la régulation économique. Les économistes savent depuis plus d'un siècle ce que nie l'idéologie libérale: l'économie capitaliste ne s'autorégule pas. Cette crise montre par ailleurs la fragilité d'une économie mondialisée dont la plupart des filières sont en étroite interdépendance.

Au-delà des conséquences incalculables et souvent dramatiques qu'elle a sur l'ensemble des populations, cette crise est également un révélateur extraordinaire et un objet de réflexion privilégié pour l'économie sociale. Elle révèle combien une partie de l'économie sociale est, qu'elle le veuille ou non, dépendante du système de régulation capitaliste. Fréquemment, l'insertion dans l'économie dominante est vécue par les entreprises d'économie sociale - surtout par de grandes coopératives et mutuelles intervenant dans un environnement hyperconcurrentiel - comme un facteur de consolidation. Dans certains cas, elle se présente même comme la condition de leur survie. La crise financière nous rappelle que cette insertion peut également être un facteur de fragilisation, voire de disparition de l'entreprise d'économie sociale ou de l'entreprise tout court.

C'est pourquoi cette crise révèle également l'urgence de réhabiliter et de rénover les autres formes économiques: économie "proprement privée ", selon le terme de Georges Fauquet - exploitations familiales agricoles, artisanat, commerce de détail -, économie publique et, enfin et surtout, économie sociale. Rénover, parce que ces économies ne peuvent renforcer leur poids qu'en se donnant de nouveaux moyens et en trouvant des réponses toujours nouvelles aux enjeux auxquels elles doivent faire face.

C'est donc l'occasion de lancer un appel aux entreprises d'économie sociale en les invitant à accentuer leurs investissements dans la recherche afin qu'ensemble, acteurs et chercheurs de l'économie sociale trouvent à cette crise des solutions autres que celles qui consisteraient à remettre en selle un système qu'on savait socialement profondément inégalitaire et qu'on sait désormais économiquement fragile: les deux vont de pair et le meilleur garant d'une économie durable n'est pas la rentabilité du placement financier. Ce sont les femmes et les hommes considérés à la fois comme finalité et comme responsables de l'action économique.

Ce contexte ne donne que plus de relief à ce numéro 310, qui comprend un nombre de pages d'actualités plus important que les numéros précédents, avec une nouvelle rubrique "En bref » qui s'ajoute aux «Temps forts de l'actualité " et à l'" Agenda ». Cette évolution tient à l'importance croissante des manifestations d'économie sociale en France et dans le monde. Le succès 
du Mois de l'économie sociale (qui déborde en réalité largement le mois de novembre), dans lequel les chambres régionales de l'économie sociale (Cres) sont très engagées, n’y est pas pour rien.

Trois articles se penchent sur une coopération agricole en pleine transformation. Franck Thomas étudie l'intercoopération qui se dessine à travers l'intérêt que les coopératives agricoles, et particulièrement les coopératives d'utilisation de matériel agricole (Cuma), portent aux sociétés coopératives d'intérêt collectif (Scic) et plus largement aux échanges entre producteurs et consommateurs et aux filières courtes. Pour modestes qu'elles soient, les initiatives en cours témoignent d'une évolution en profondeur des pratiques coopératives qui mérite la plus grande attention.

Maryline Filippi, Olivier Frey et René Mauget présentent une large étude des coopératives agricoles, que les évolutions de la politique agricole commune et de l'Organisation mondiale du commerce poussent à se concentrer et à s'internationaliser. Les auteurs analysent les stratégies des groupes pour répondre à ces nécessités, montrent leurs nouveaux besoins de financement et de partenariat et soulignent la complexité de la situation actuelle: la double nécessité de relancer la production pour répondre aux besoins en alimentation et de respecter les conditions d'un développement durable.

Lionel Bobot illustre un aspect de ce problème à partir de l'étude du développement mondial de Yoplait (détenu par la coopérative Sodiaal) par l'usage de la franchise, à la fois outil d'intercoopération entre grandes entreprises et facteur de transformation du statut du sociétaire, invité à devenir également actionnaire des filiales commerciales. Transformation non sans risques, puisqu'elle peut aboutir à la réduction de la rémunération de la part sociale du sociétaire, réduction " compensée " par l'augmentation des dividendes dus aux actionnaires.

Nous sommes particulièrement heureux de publier l'article d'Eric Bidet sur les raisons de "la difficile émergence de l'économie sociale en Corée du Sud ». L'auteur montre en particulier ce que l'économie doit aux données sociales et politiques, et spécialement aux structures familiales, à la religion, à l'Etat, à l'histoire. Ce parcours de la pensée est trop peu fréquenté et l'auteur nous convainc de l'intérêt, pour mieux l'appréhender, de resituer plus souvent l'économie sociale dans un contexte non économique.

Olivier Chaïbi ressort de l'anonymat Jules Lechevalier (1806-1862). Sa passionnante contribution éclaire les liens, plus étroits qu'on ne le pense généralement, entre les pensées de Saint-Simon, de Fourier et de Proudhon. Il précise la relation - fondatrice en économie sociale - entre théories et pratiques et montre l'intérêt spécifique des biographies, en particulier dans notre champ d'intérêt: il existe d'autres Jules Lechevalier à découvrir!

Ce numéro est le premier réalisé depuis dix ans sans Sylvie Mosser. Jordane Legleye lui a succédé cet été. Au nom des rédacteurs associés, je lui souhaite la bienvenue. 